\title{
Relativistic jets: Physics and simulations
}

\author{
John F. Hawley \\ Department of Astronomy, University of Virginia Charlottesville, VA 22904 USA \\ email: jh8h@virginia.edu
}

\begin{abstract}
Jets are one of the more dramatic and visible manifestations of black hole accretion. It is becoming increasingly accepted that magnetic fields underlie the mechanism behind the launching of relativistic jets. At the same time it is now appreciated that the fundamental driving mechanism behind accretion is due to magnetohydrodynamical processes and the stresses they engender. Global disk simulations in full general relativity have begun to reveal the radial dependence of disk stresses and the intimate connections between accretion and jet-launching. This talk will review the issues involved, the recent progress that has been made, and the challenges that remain.
\end{abstract}

Keywords. accretion, accretion disks, black hole physics, MHD

\section{Introduction}

The topic of this symposium is "Jets at All Scales" and, indeed, jets are seen on many scales and in a wide variety of astrophysical objects. Protostellar systems, binary stars, active galactic nuclei, quasars, neutron stars and white dwarfs, all have demonstrated the ability to generate jets. The ubiquity of the jet phenomenon argues that jets do not require particularly exotic circumstances in which to form. Jets appear to be the consequence of accretion, rotation, and magnetic fields (Livio 2000). When the jet's energy is substantial and the velocities relativistic, the jet most likely originates from a black hole system. After all, the environment near a black hole is nothing if not relativistic!

The dominant paradigms for jet launching follow the ideas outlined in the influential papers of Blandford \& Znajek(1977) and Blandford \& Payne(1982). In the BlandfordPayne (BP) model the power for the jet comes from the accretion disk. A large-scale poloidal magnetic field is anchored in and rotates with the disk. If the fieldlines are angled outward sufficiently with respect to the disk, there can be a net outward force on the matter. As matter is accelerated along the rotating fieldlines, its angular momentum increases still further, increasing the acceleration and driving an outflow.

In the $\mathrm{BP}$ model the energy driving the jet derives from the rotational energy in the accretion disk. But the central star in an accreting system can also rotate and drive a jet. Even a black hole, which lacks a solid surface, can do so. In the Blandford-Znajek (BZ) model the jet is powered by the rotating space-time of a black hole. In this model, radial magnetic field lines pass through the ergosphere and the horizon of the hole. Frame dragging by the rotating hole leads to the creation of toroidal field and hence a Poynting flux. The key to the BZ process is that within the ergosphere it is possible to have an electromagnetic flux with negative energy at infinity. This negative energy flux enters the hole, thereby reducing both the hole's mass-energy and angular momentum. The outgoing Poynting flux can have substantial positive energy derived from the black hole. 


\section{Magnetic Fields and Accretion}

The common thread in both the BP and the BZ models is the presence of a large-scale poloidal field that can tap into the rotational energy of the system, either that in the disk or the black hole. The notion that jets are fundamentally a magnetic phenomenon fits in nicely with our emerging overall understanding of accretion itself. Accretion depends on the removal of angular momentum from fluid elements by some internal stress. Although differential rotation is linearly stable to hydrodynamic perturbations by the Rayleigh criterion, it is destabilized by the presence of a magnetic field (Balbus \& Hawley 1991, 1998; Balbus 2003). The magnetorotational instability (MRI) leads to turbulence and Maxwell and Reynolds stresses. From linear theory we know that the MRI is present as long as the field is relatively weak which, for an accretion disk, means subthermal, i.e., has a plasma $\beta=P / P_{\text {mag }}$ greater than 1 . The maximum growth occurs when $\left(\mathbf{k} \cdot \mathbf{v}_{\mathrm{A}}\right)^{2}$ is comparable to $\Omega^{2}$. This corresponds to a wavelength that is about the distance an Alfvén wave travels in an orbit. All orientations of magnetic field can be unstable, including nonaxisymmetric modes involving the toroidal field.

The presence of the linear MRI provides the means to destabilize the otherwise laminar orbital motion of the gas. The properties of the resulting fully developed MHD turbulence are not accessible to analytic theory, however, and we have to rely upon simulations to gain insight.

\section{Simulations}

For the most part, the theory of jet formation and black hole accretion is based on somewhat idealized and parameterized models that have characteristics consistent with the observed phenomena. One would prefer to begin with a very general set of welldefined equations and from them develop a model from first-principles. We are still a long way from that ideal, but we do know one thing: the equations of MHD plus gravity provide a minimal set of conservation equations that contain sufficient physics to describe the basic dynamics of the accretion process and of jet formation.

\subsection{Local Shearing Boxes}

The most detailed simulations of the MRI-driven turbulence that drive accretion have been done in a local approximation known as the shearing box (Hawley et al. 1995). These shearing box simulations have revealed a number of important properties of magnetized accretion. First, the MRI leads to turbulence which produces angular momentum transport. The important component of the stress tensor is

$$
\tau_{r \phi}=-B_{r} B_{\phi} / 4 \pi+\rho v_{r} \delta v_{\phi}
$$

where the first term is the Maxwell stress and the second is the Reynolds stress. In MRI turbulence the Maxwell stress always exceeds the Reynolds stress by about a factor of 4 , and the total $r \phi$ component of the stress is proportional to the magnetic pressure, $\tau_{r \phi}=\alpha_{\mathrm{mag}} P_{\mathrm{mag}}$, where $\alpha_{\mathrm{mag}}$ is the constant of proportionality. Blackman, Penna \& Varnière (2008) summarized the results from a large number of shearing box simulations in the literature and showed that quite generally $\alpha_{\text {mag }}$ is nearly constant across a wide range of simulations, with a value $\sim 0.4-0.5$. The problem for constructing accretion disk models is that we still don't know what sets $P_{\text {mag }}$ within a disk, except that it is likely to be subthermal, i.e., $\beta=P_{\text {gas }} / P_{\text {mag }}>1$. Note that if one uses the conventional Shakura \& Sunyaev(1973) $\alpha$ value to characterize the stress, a specific value of $\alpha$ implies a magnetization of $\beta=\alpha_{\mathrm{mag}} / \alpha$. Typical $\beta$ values in shearing box simulations are $\sim 10-100$, corresponding to $\alpha$ values $\sim 0.1-0.005$. 
One of the defining characteristics of the $\alpha$ disk model is that the same stress that accounts for the angular momentum transport also determines the local heating (Balbus \& Papaloizou 1999). This requires that the orbital energy released by the stress go directly into the energy of the MHD turbulence, and cascade to small scales where it is thermalized promptly. This need not necessarily be the case; if energy is transported by waves or by the motion of strong fields (for example, field buoyantly rising into a corona) rather than rapidly dissipating as heat, things become nonlocal. This may happen under some circumstances, but shearing box simulations (e.g., Simon, Hawley \& Beckwith 2009) find that turbulent dissipation occurs rapidly, on a timescale of order $\Omega^{-1}$, roughly a turnover time of the largest scale turbulent eddies and consistent with the requirements of the $\alpha$ model. In the radiative models of Hirose et al. (2009) dissipation is local and the bulk of the energy is carried out of the disk by radiation. Only a small fraction is carried by magnetic flux.

One great advantage of the local model compared to global simulations is that is is easier to add more complex physics and to employ greater resolution. The most realistic shearing box simulations done to date are those that include radiation transport (e.g., Turner 2004; Hirose, Krolik \& Stone 2006). One set of such simulations carried out by Hirose, Krolik \& Blaes (2009) who answered a long-standing question in disk modeling. If one adopts the $\alpha$ formulation for the stress, radiation-dominated disks become thermally unstable (Lightman \& Eardley 1974; Shakura \& Sunyaev 1976). Essentially the stress, and hence disk heating, increase faster than the radiative cooling rate. This thermal instability goes away if the Shakura-Sunyaev stress formula is modified so that the stress is proportional to the gas pressure alone rather than the total pressure. Then an increase in radiation pressure does not lead to increased heating. It has been long suspected that instabilities such as the thermal instability are simply artifacts of the $\alpha$ prescription (Pringle 1981). This seems to be the case, at least for the local thermal instability. Hirose et al. (2009) demonstrated that although the stress is, on average, proportional to the total pressure, there is no thermal instability in radiation pressure supported disks. The reason is that stress determines the pressure, not the other way around. An increase in stress will lead to an increase in pressure, but an increase in pressure does not necessarily lead to an increase in magnetic energy and hence stress. Although this result rules out the local thermal instability, various global instabilities remain possible. The shearing box's ability to investigate global properties is quite restricted.

\subsection{Global Simulations - Jet Production}

One area where local simulations are of particularly limited use is in modeling jet formation; global simulations are essential. Some of the questions that might be addressed by simulations include:

- Does a particular jet model in fact produce a stable, sufficiently energetic jet?

- What magnetic field configurations and strengths lead to jet formation?

- Under what circumstances does an accreting system develop the required magnetic fields?

For example, the viability of the BP process has been demonstrated by following the evolution of a disk embedded in an initial vertical field (see the review by Pudritz et al. 2007). The questions are whether the necessary field configurations are stable over the long term, and whether they can arise naturally as a result of the accretion process.

For black holes, global simulations have produced reasonably powerful and stable jets consistent with the BZ mechanism, beginning from simple initial conditions. The key to jet formation is to establish the right kind of magnetic field, here a large-scale poloidal field along the axis of the black hole. The first GR simulations (De Villiers et al. 2005; 
Hawley et al. 2006; McKinney 2006) start with dipole field loops contained entirely within an initial orbiting gas torus. Early in the simulation, inflow drags the inner part of these loops down to the hole creating an extended radial field. Relative shear along this field creates toroidal field that grows in amplitude. This drives a vertical expansion of the field generating a magnetic tower (Lynden-Bell 2006; Kato et al. 2004) which eventually resolves itself as a large-scale dipole field embedded on the black hole. The rotation of the black hole produces a Poynting flux moving outward at relativistic velocities. The power of the Poynting flux jet is determined by the spin of the black hole and the strength of the magnetic field. In the simulations the resulting jet power matches well with the predictions of the BZ model (McKinney \& Gammie 2004; McKinney 2005). The Lorentz factor $\Gamma$ in the jet depends on the mass loading. The Poynting flux jet has very little mass within it; the angular momentum within the gas is too large to enter the axial funnel. For numerical stability the simulations must impose a density floor and this can control the mass density within the funnel. However, jets with $\Gamma>10$ have been produced (e.g., McKinney 2006).

Hawley \& Krolik (2006) examined a series of simulations that employed black holes with rotations ranging from retrograde with $a / M=-0.9$ to prograde with $a / M=0.99$. They found that for prograde models with $a / M \sim 0.9$ and above the jet energy can exceed the nominal energy associated with accretion as determined by the binding energy of the last stable orbit associated with that black hole spin. Both the increase in spin and an increase in the field strength within the funnel account for the strong increase in jet energy. For the retrograde model the sign of the angular momentum in the jet is consistent with that of the hole rather than the disk, establishing that the source of rotation is, indeed, the hole. The jet energy is greater than the nominal energy of accretion for the retrograde disk, but considerably lower than the power in the prograde $a / M=0.9$ jet. This result is not consistent with the model discussed in the talk by David Meier (these proceedings) wherein the retrograde holes have the strongest jets. In these simulations the process producing the magnetic tower that leads to the funnel field is largely insensitive to the black hole spin. The depth of the last stable orbit helps to set the funnel field's strength, and once established that field is relatively constant. Ultimately the issue depends on the processes by which Nature loads a black hole with poloidal field, and many uncertainties remain in this topic.

In the simulations, unbound jets with significant mass flux arise only outside the centrifugal boundary surrounding the axis. These jets are accelerated by fields that remain within the accretion flow; pressure forces push gas up against the centrifugal barrier and outward, resulting in an unbound mass flux and an outgoing velocity of about $\sim 0.5 c$ (De Villiers et al. 2005; Hawley et al. 2006). This is the only jet seen in simulations with nonrotating Schwarzschild holes.

These simulations succeed in producing a jet, and suggest several questions for further investigation. First, when will the necessary field get established on the black hole? Jet formation requires a consistent sense of vertical field to be brought down to the event horizon. This readily occurs for simulations that begin with a large dipole loop. But simulations by Beckwith et al. (2008) found that much weaker funnel fields result from quadrupolar field initial conditions, and no funnel field at all develops for toroidal field initial conditions. A strong jet seems to require a net vertical field in the disk midplane whose sign remains consistent for at least an inner-disk inflow time. In simulations with an initial quadrupole field, a funnel field can be established, but the presence of a current sheet in the corona between the black hole's axis and the equator makes conditions favorable for reconnection and field loss. In the dipole case, the current sheet is located at the equator inside the disk and the funnel field is relatively insulated against reconnection. 
For purely toroidal field initial conditions there is MRI-driven turbulence, but the poloidal field coherence length tends to be relatively short. There is insufficient field amplification to create a magnetic tower and thereby load the funnel with field.

Assuming that a poloidal funnel field is established, what determines the strength of that field? Within the disk the basic requirements of the MRI suggest that the field will be subthermal, possibly with $\beta \sim 10-100$. If the funnel field were of comparable strength it would be difficult to drive a powerful jet, particularly for cool, thin disks. However, the field strength in the funnel may not be so limited. In the simulations, the magnetic pressure within the funnel is more or less comparable to the total pressure in the inner disk, and the field is correspondingly stronger. While this result seems entirely reasonable, it remains to be seen if it holds under all circumstances.

\subsection{Radial Advection of Field:}

Jets require the development of a large-scale poloidal field passing through the disk, the black hole, or both. How does such a field arise? Since the disk is fundamentally magnetic, it is possible that the MRI can act as a dynamo to generate the required field. In fact, stratified shearing box simulations have found that a mean toroidal field can be generated at the disk midplane and buoyantly rise out, to be replaced by a mean toroidal field of the opposite sign. This happens on 10 orbit timescale and is consistent with a simple $\alpha-\Omega$ dynamo (Brandenburg et al. 1995; Stone et al. 1996; Hirose et a. 2006; Guan 2009; Shi et a. 2010; Gressel2010; Davis et al. 2010; Simon et al. 2010). The resulting field does not appear, however, to have the properties required for jets.

An alternative way to obtain a large-scale net field in the near-hole region is by bringing net field in from large radius. A field that is very weak far from the hole can be strongly amplified as it is concentrated by accretion. The issue is whether the rate at which the field diffuses through the matter exceeds the rate at which it is accreted (van Ballegooijen 1989; Lubow et al. 1994; Heyvaerts et al. 1996). This question is usually framed in terms of a competition between an effective turbulent viscosity, $\nu_{t}$, and an effective magnetic diffusivity, $\eta_{t}$. The conventional assumption is that the turbulent viscosity will be comparable to diffusivity, and field advection will be inhibited. Local simulations by Guan \& Gammie (2009) seem to support this viewpoint. Guan \& Gammie examined the rate at which a sinusoidal vertical flux distribution decayed within MRI turbulence and found a diffusion time on order several tens of $\Omega^{-1}$, comparable to the accretion time.

Beckwith et al. (2009) carried out global simulations to directly address the question of whether net vertical flux can be advected inward by MRI-driven turbulence. In this simulation an initial isolated, uniform vertical field passes through a gas torus. At the beginning of the simulation the field in the low density region outside of the disk expands. Within the disk the field is unstable to the MRI. Turbulence develops on an orbital timescale and the disk begins to accrete. While the magnetic flux does diffuse somewhat within the disk, it is not carried in efficiently with the accretion flow. In fact, much of the initial vertical flux within the disk remains near its initial location. Nevertheless, the black hole rapidly acquires a net dipole field. Instead of being brought in by the disk, this field is carried in by infall of low density and low angular momentum material in the corona surrounding the disk. These results suggest two points. First, field is most effectively transported by an inflow driven not by turbulent stresses, but by large-scale magnetic torques operating in low density gas (e.g., Rothstein \& Lovelace 2008). Second, net flux is a global, not a local concept. Here, the acquisition of a net dipole on the hole requires both field to be carried down to the black hole, and reconnection across the equator to change the global topology. 


\subsection{The Spin of the Hole}

As several talks at this Symposium have indicated, there are important issues related to the spin of black holes, including whether or not we can measure black hole spin from observed disk or jet properties, and whether there is any observational evidence for the role of black hole spin in powering jets (see, e.g., the talk by Elena Gallo).

Current methods of black hole spin measurement, including both continuum fitting and $\mathrm{Fe} \mathrm{K} \alpha$ line fitting, rely on knowledge of the underlying disk and its inclination angle. These procedures rely on the assumption that the disk's inner edge coincides precisely with the innermost stable circular orbit (ISCO) which is itself precisely determined by $a / M$. In fact, the situation is likely to be a bit more complex than that. What constitutes a disk's inner edge, for example, depends on the property being examined (Krolik \& Hawley 2002). There will be a location where from which the last photons emerge (the radiation edge, relevant to continuum fitting), a location where the optical depth drops below the point where photons can be reflected (the reflection edge, relevant to Fe $\mathrm{K} \alpha$ line modeling), a location where turbulent flow is replaced by laminar inflow (the turbulence edge), and the place where the internal stress goes to zero (the stress edge). The standard model (Novikov \& Thorne 1973) assumes that all these edges are located at the ISCO. This may be problematic; magnetic fields can exert force over extended distances. Gammie(1999) and Krolik(1999) showed that, in principle, significant magnetic stress can be present within the ISCO, even for cold, thin disks. Global simulations have found that significant magnetic stress can continue right through and inside the ISCO (Hawley \& Krolik 2001; Reynolds \& Armitage 2001; Hawley \& Krolik 2002; Machida \& Matsumoto 2003; Gammie et al. 2004; Krolik et al. 2005).

The disks in these first simulations were all modestly thick, with $H / R \sim 0.15$. Because the magnetic stress might be a function disk temperature and hence $H$ at the ISCO, simulations were carried out that included direct regulation of disk temperature and thickness. Noble, Krolik \& Hawley (2009) used a simple cooling function to compute a disk with $H / R \sim 0.1$ accreting into an $a / M=0.9$ hole and found enhanced stress that led to a $6 \%$ increase in luminosity at infinity over the conventional value. Shafee et al. (2008) found a reduced (but still nonzero) stress inside the ISCO for thin disks, as did Penna et al. (2010), with the net specific angular momentum accreted into the hole reduced by only a few percent below the ISCO value. A systematic study by Noble et al. (2010) considered different $H / R$ values accreting into a Schwarzschild black hole and found that functionally the stress continues to rise through the ISCO, dropping to zero only right outside of the black hole horizon. The Maxwell stress showed no dependence on $H$, but the Reynolds stress at the ISCO did decline with disk thickness. Beckwith et al. (2008) found that the initial field topology could influence the stress at the ISCO. In particular, a simulation that began with a purely toroidal initial field barely had any enhanced stress at the ISCO. Penna et al. (2010) pointed out that the differences in stress levels seen in various thin disk simulations could be due to different initial magnetic field topologies and strengths.

Where does this leave us currently? It is clear that magnetic fields can increase the efficiency of accretion through enhanced stress at the ISCO, but the precise amount depends on both physical and numerical factors. The simple "zero stress at the ISCO" assumption is not universally applicable.

Simulations also have implications for the evolution of the black hole's spin if it is being determined by accretion. Spin evolution was considered by Thorne (1974) who showed that although accretion would tend to spin up the hole to its physical limit, the capture of photons from the disk, preferentially with retrograde orbits, limits the spin-up to 
$a / M=0.998$. Simulations have found that the upper limit could be considerably smaller for magnetized accretion (Gammie et al. 2004; McKinney \& Gammie 2004; Krolik et al. 2005; Beckwith et al. 2008). Magnetic fields threading the horizon can carry angular momentum to infinity as the hole captures Alfvén waves with retrograde angular momentum. In the simulations of McKinney \& Gammie (2004) the angular momentum flux into holes with different spins was measured and the transition point between net spin-up and spin-down was at $a / M \sim 0.93$. That number is specific to this set of simulations, but if this general idea holds then the most rapidly spinning (and hence most energetic) holes might be rare or nonexistent in Nature.

\section{Summary}

Simulations are currently playing an important role in investigating the physics of black hole accretion, from the stresses within the disks to the formation of jets. Local shearing box simulations have demonstrated that the MRI leads to turbulence that accounts for angular momentum transport in disks. While the stress is related to the pressure, it is not strictly determined by it. Rather, the stress is directly proportional to the magnetic pressure. What determines the magnetic pressure under different conditions remains a topic of continuing investigation.

Simulations have successfully demonstrated the creation and maintenance of Poynting flux jets from spinning black holes. These jets require a dipole field within the axial funnel of the hole. The manner in which such a field might generally be created and how it would be sustained remain open questions. It may be that net flux can be brought in from large radius by some aspect of the accretion process. It is also possible that the process is stochastic, simply dependent upon a random build up from field brought in by accretion. If so, jets would be intermittent on accretion timescales.

Simulations are providing new details regarding the properties of the near-hole region of the accretion disk. Magnetic stresses can operate through the ISCO, potentially altering the accretion efficiency and luminosity. By capturing Alfvén waves with retrograde angular momentum, magnetic fields have the ability to limit $a / M$ in black holes whose spin is determined by accretion.

We have long known that gravity is a potential source of energy, and that angular momentum leads to disks that can efficiently extract and radiate that energy. But it turns out that it is the magnetic field that really makes things lively!

\section{Acknowledgements}

This work was supported by NASA grant NNX09AD14G and NSF grant AST-0908869. I thank collaborators Steve Balbus, Kris Beckwith, Jean-Pierre De Villiers, Xiaoyue Guan, Andrew Hamilton, Julian Krolik, Scott Noble, Jeremy Schnittman, and Jacob

Simon. Computational resources were supplied by the TeraGrid, supported by the National Science Foundation.

\section{References}

Balbus, S. A. 2003, Ann. Rev. Astron. Astrophys., 41, 555

Balbus, S. A. \& Hawley, J. F. 1991, ApJ, 376, 214

- 1998, Rev. Mod. Phys., 70, 1

Balbus, S. A. \& Papaloizou, J. C. B. 1999, ApJ, 521, 650

Beckwith, K., Hawley, J. F., \& Krolik, J. H. 2008, ApJ, 678, 1180

- 2009, ApJ, 707, 428 
Blackman, E. G., Penna, R. F., \& Varnière, P. 2008, New Astron., 13, 244

Blandford, R. D. \& Payne, D. G. 1982, MNRAS, 199, 883

Blandford, R. D. \& Znajek, R. L. 1977, MNRAS, 179, 433

Brandenburg, A., Nordlund, A., Stein, R. F., \& Torkelsson, U. 1995, ApJ, 446, 741

Davis, S. W., Stone, J. M., \& Pessah, M. E. 2010, ApJ, 713, 51

De Villiers, J., Hawley, J. F., Krolik, J. H., \& Hirose, S. 2005, ApJ, 620, 878

Gammie, C. F. 1999, ApJ, 522, L57

Gammie, C. F., Shapiro, S. L., \& McKinney, J. C. 2004, ApJ, 602, 312

Gressel, O. 2010, MNRAS, 405, 41

Guan, X. 2009, PhD Thesis, Univ. of Illinois

Guan, X. \& Gammie, C. F. 2009, ApJ, 697, 1901

Hawley, J. F., Gammie, C. F., \& Balbus, S. A. 1995, ApJ, 440, 742

Hawley, J. F. \& Krolik, J. H. 2001, ApJ, 548, 348

- 2002, ApJ, 566, 164

- 2006, ApJ, 641, 103

Heyvaerts, J., Priest, E. R., \& Bardou, A. 1996, ApJ, 473, 403

Hirose, S., Krolik, J. H., \& Blaes, O. 2009, ApJ, 691, 16

Hirose, S., Krolik, J. H., \& Stone, J. M. 2006, ApJ, 640, 901

Kato, Y., Mineshige, S., \& Shibata, K. 2004, ApJ, 605, 307

Krolik, J. H. 1999, ApJ, 515, L73

Krolik, J. H. \& Hawley, J. F. 2002, ApJ, 573, 754

Krolik, J. H., Hawley, J. F., \& Hirose, S. 2005, ApJ, 622, 1008

Lightman, A. P. \& Eardley, D. M. 1974, ApJ, 187, L1

Livio, M. 2000, in American Institute of Physics Conference Series, edited by S. S. Holt, \&

W. W. Zhang, vol. 522 of American Institute of Physics Conference Series, 275

Lubow, S. H., Papaloizou, J. C. B., \& Pringle, J. E. 1994, MNRAS, 267, 235

Lynden-Bell, D. 2006, MNRAS, 369, 1167

Machida, M. \& Matsumoto, R. 2003, ApJ, 585, 429

McKinney, J. C. 2005, ApJ, 630, L5

- 2006, MNRAS, 368, 1561

McKinney, J. C. \& Gammie, C. F. 2004, ApJ, 611, 977

Noble, S. C., Krolik, J. H., \& Hawley, J. F. 2009, ApJ, 692, 411

- 2010, ApJ, 711, 959

Novikov, I. D. \& Thorne, K. S. 1973, in Black Holes (Les Astres Occlus), edited by C. De Witt (Gordon and Breach, New York), 343

Penna, R. F., McKinney, J. C., Narayan, R., Tchekhovskoy, A., Shafee, R., \& McClintock, J. E. 2010, MNRAS, 1216

Pringle, J. E. 1981, Ann. Rev. Astr. Astrophs., 19, 137

Pudritz, R. E., Ouyed, R., Fendt, C., \& Brandenburg, A. 2007, in Protostars and Planets V, edited by B. Reipurth, D. Jewitt, \& K. Keil, 277

Reynolds, C. S. \& Armitage, P. J. 2001, ApJ, 561, L81

Rothstein, D. M. \& Lovelace, R. V. E. 2008, ApJ, 677, 1221

Shafee, R., McKinney, J. C., Narayan, R., Tchekhovskoy, A., Gammie, C. F., \& McClintock, J. E. 2008, ApJ, 687, L25

Shakura, N. I. \& Sunyaev, R. A. 1973, A\&A A, 24, 337

- 1976, MNRAS, 175, 613

Shi, J., Krolik, J. H., \& Hirose, S. 2010, ApJ, 708, 1716

Simon, J. B., Hawley, J. F., \& Beckwith, K. 2009, ApJ, 690, 974

- 2010, ApJ, submitted. Astro-ph 1010.0005

Stone, J. M., Hawley, J. F., Gammie, C. F., \& Balbus, S. A. 1996, ApJ, 463, 656

Thorne, K. S. 1974, ApJ, 191, 507

Turner, N. J. 2004, ApJ, 605, L45

van Ballegooijen, A. A. 1989, in Accretion Disks and Magnetic Fields in Astrophysics, edited by G. Belvedere, vol. 156 of Astrophysics and Space Science Library, 99 


\section{Discussion}

NEILSEN: If you are suggesting that spin measurements are only of modest reliability beacuse of stress inside the ISCO, could you comment on recent simulations that show that this stress is due to stretching of the magnetic field, and that because the flow is laminar there is no dissipation?

HAWLEY: Additional stress at the ISCO is only one uncertainty in spin measurement and probably not the largest. If stess is due to the "field stretching" work is still done and energy is transferred. Nonzero stress at the ISCO has a significant effect outside of the ISCO (compared to the zero stress assumption). Of course, what will be seen at infinity depends on many factors including dissipation, emission, photon capture by the black hole, etc.

YUAN: Why the magnetic field dissipation timescale is the orbital time if it's intrinsically magnetic reconnection?

HAWlEY: Dissipation is occurring through a turbulent cascade. The eddy turnover time is of the order of $\Omega^{-1}$ in the turbulence. In simulations we assume thermalization at the grid scale. Detailed studies of MHD turbulence are required to know.

KoIDE: How large is the magnetic Reynolds number in your GRMHD simulations? If you consider the numerical resistivity, it depends on the Mach number of the simulations?

HAWLEY: In these simulations, magnetic reconnection occurs at the grid scale. Numerical reconnection is not directly analogous to Ohmic resistivity, so it is difficult to assign an specific Reynolds number. Simon et al. (ApJ 2009) measured an effective magnetic Reynolds number for the Athena code and obtained $R_{n} \sim 8000$ for 128 zone resolution with the $R_{n}$ value proportional to $\Delta x^{-2}$.

MeIER: The most reliable $\mathrm{BH}$ spin determination are $>0.98$. Also, Fender, Gallo, \& Belloni (2010) studied several $\mu$ QSRs and concluded that the BZ process is not active in these systems. Please comment.

HAWLEY: I suspect that the spin measurements are not reliable to the requested level. Also the BZ process dependes not just on the black hole spin, but also on the magnetic field topology and strength.

PE'ER: Given that MRI is most effective for weak B-fields, what is the typical ratio of Poynting energy to matter (kinetic) energy in the jet?

HAWLEY: Although the MRI field has $B^{2}<P$ in the disk, the funnel field driving the Poynting flux of order $B^{2}=P$ (disk), that is overall pressure equilibrium. Under those circumnsances, we have found that Poynting flux energy can be comparable to the disk luminosity for large black hole spins. 